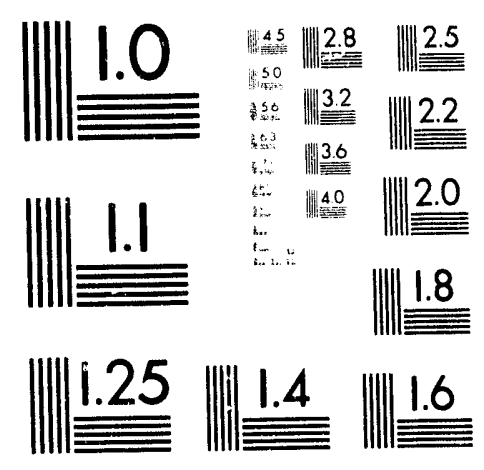



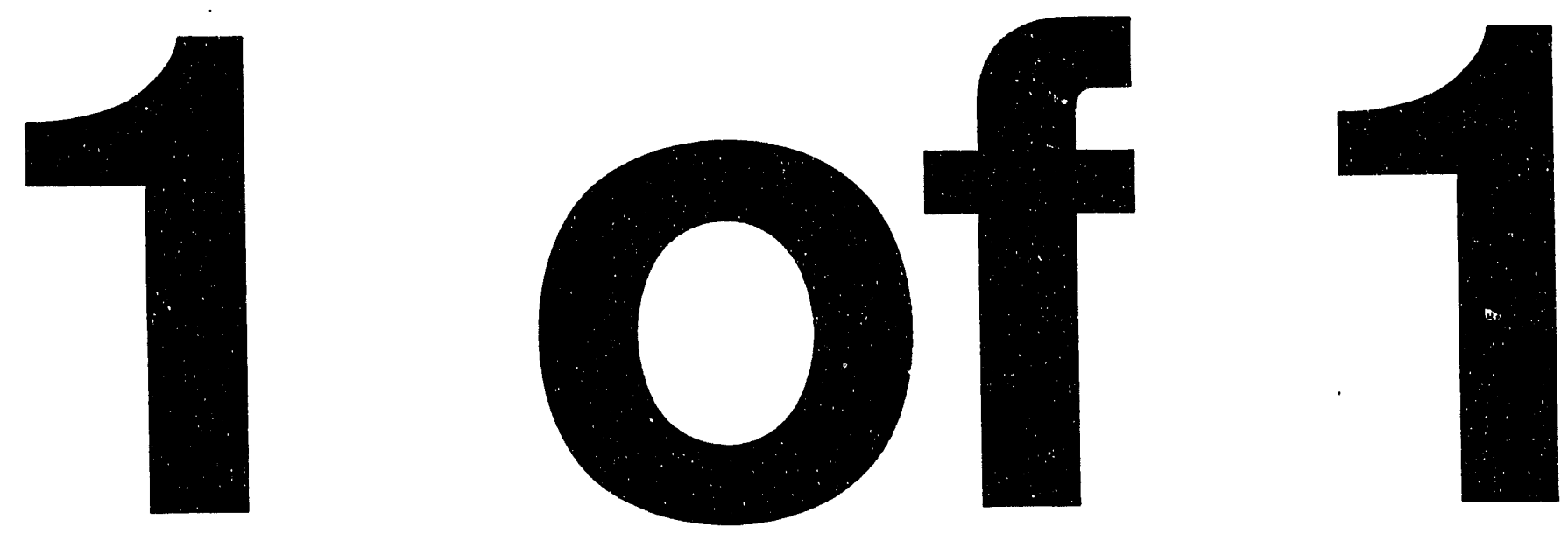


\title{
$\cos (-93045-41$
}

UCRL-JC-113802

PREPRINT

\section{PHOTOELECTRON HOLOGRAPHY OF PLATINUM (111)}

\author{
Barry L. Petersen \\ L.J. Terminello \\ J.J. Barton \\ D.A. Shirley
}

This paper was prepared for submittal to the 1993 Spring Materials Research Society Conference Proceedings San Francisco, CA

April 12-17, 1993

April 1993

This is a preprint of a paper intended for publication in a joumal or proceedinga. Since changes may be made before publication, this preprint is made available with the understanding that it will not be cited or reproduced without the permiscion of the author.

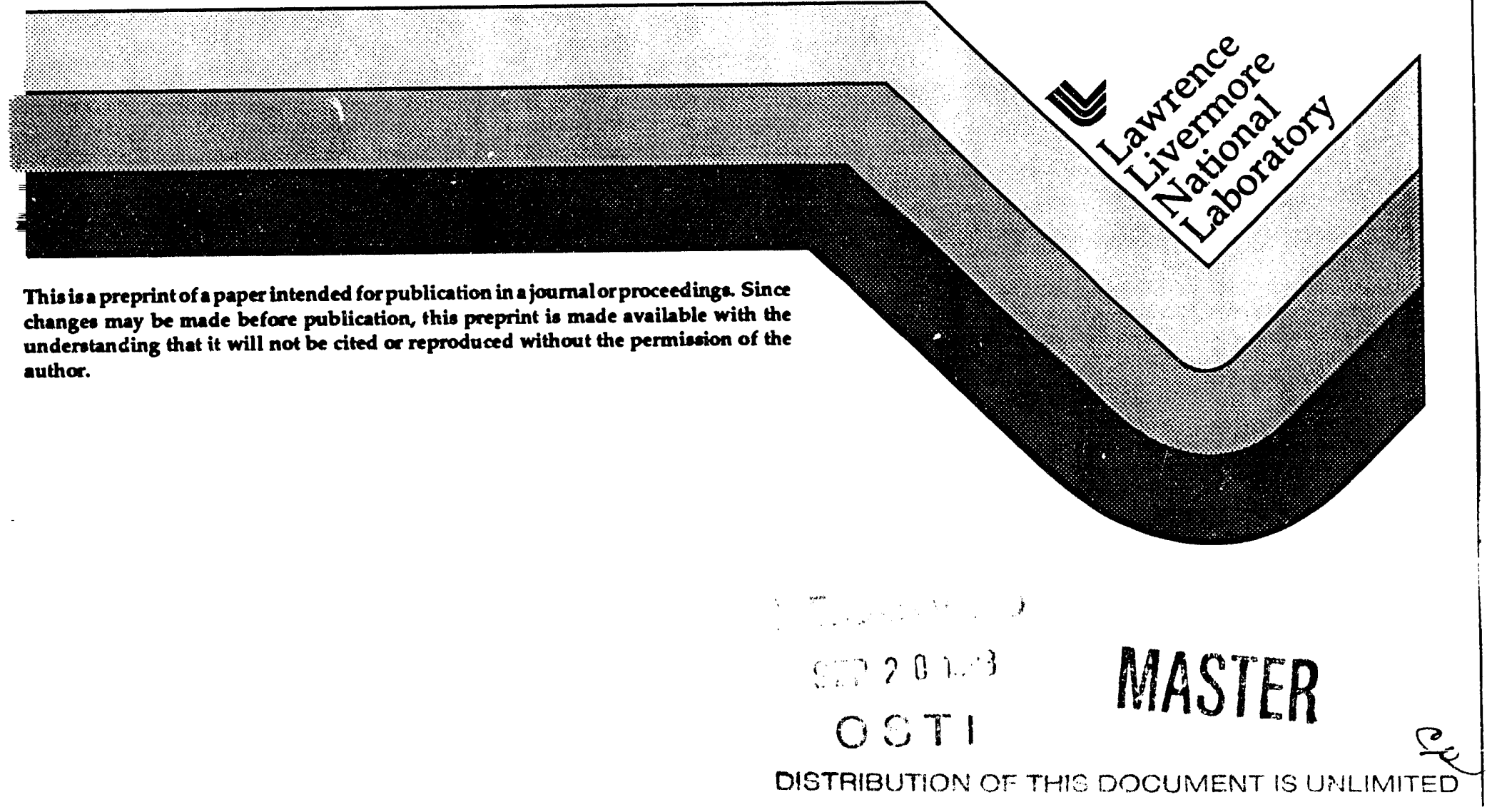




\section{DISCLAIMER}

This document was prepared as an account of work sponsored by an agency of the United States Government. Neither the United States Government nor the University of California nor any of their employees, makes any warranty, express or implied, or assumes any legal liability or responsibility for the accuracy, completeness, or usefulness of any information, apparatus, product, or process disdosed, or represents that its use would not infringe privately owned rights. Reference herein to any specific commercial products, process, or service by trade name, trademark, manufacturer, or otherwise, does not necessarily constitute or imply its endorsement, recommendation, or favoring by the United States Government or the University of California. The views and opinions of authors expressed herein do not necessarily state or reflect those of the United States Government or the University of Califormia, and shall not be used for advertising or product endorsement purposes. 
BARRY L. PETERSEN 1 , L.J. TERMINELLO ${ }^{2}$, J.J. BARTON ${ }^{3}$, AND D.A. SHIRLEY 4

'Lawrence Berkeley Laboratory, M/S 2-300, 1 Cyclotron Rd., Berkeley, CA 94720

2Lawrence Livermore National Laboratory, M/S L-357, Livermore, CA 94550

3IBM T.J. Watson Research Center, Yorktown Heights, NY 10598

${ }^{4}$ Pennsylvania State University, Room 114 Kern Graduate Building, University Park, PA 16802

\section{ABSTRACT}

Platinum atoms near a (111) single-crystal face have been imaged using photoelectron holography. Electron angular intensity patterns were collected at equally spaced wavenumbers from $6 \AA^{-1}$ to $12 \AA^{-1}$. Images of atoms near expected atomic positions are obtained from singlewavenumber analyses over the range of the data set. Positions are detected further from the emitter than we have seen previously, and symmetry assumptions are not required. We have also adopted a three dimensional means of representing the data in order to help understand the results. Twin image suppression and artifact reduction in the holographically reconstructed data set are obtained when images at different wavenumbers are correctly phase-summed. We are assessing the capability of the technique for rendering true three-dimensional structural information for unknown systems.

\section{INTRODUCTION}

In the search for direct methods for structural determination of surfaces, photoelectron holography ${ }^{1,2}$ shows great promise as a general technique for exploring the environment around a given atom. Because of the inherent limited resolution of the technique, precise structural determinations may be difficult to achieve, however, directions and approximate distances of neighboring atoms in a surface may be quickly deduced without a priori knowledge of the system. The method also has all of the advantages of the chemical specificity of photoelectron diffraction. When combined with multiple-scattering analyses, this technique may achieve the goal of obtaining accurate and reliable surface structural information.

Essentially, photoelectron holography involves the formation of a 3-dimensional image of atomic positions surrounding a chosen atom in an ordered crystal or surface system. High energy photons are used to emit a photoelectron wave from a core level of the atom. The image is obtained by mathematical reconstruction of the interference pattern created from the combination of the original wave and that same wave scattered from surrounding atoms. This reconstruction involves a two-dimensional Fourier transform of the momentum-space map phased for the particular vertical distance of interest. This method has already been applied to several systems $3-7$, and has shown promise as a useful technique for determination of local structure around atoms in bulk substrates and adsorbates on ordered surfaces. In addition, methods of image correction have also been suggested which could potentially aid in experimental applications ${ }^{8-11}$. However, improvement of the technique continues, and this paper describes an extension of that study using a new substrate, the (111) surface of platinum.

In this work, we further explore the capabilities of photoelectron holography as a structuraliy useful technique. A simple fcc crystal was chosen as a well-understood test case which could be used to carefully deduce the type of information which could be extracted from these experiments. The main emphases of this endeavor include reduction of transform artifacts, symmetry without averaging, and positions of atoms further from the emitter than seen previously. We also conduct a multiple wavenumber analysis in which we show the expected reduction of twinning and transform artifacts and improvement of Z-resolution.

\section{EXPERIMENT}

The work described here was conducted in bulk platinum using a $\mathrm{Pt}(111)$ single crystal surface. The crystal was cleaned by sputtering in $1 \times 10^{-5}$ torr argon for $1 \mathrm{hr}$ followed by 
annealing by resistive heating for three minutes at $\sim 850^{\circ} \mathrm{C}$. The cleanliness of the sample was assessed from the photoelectron spectrum of platinum. Initial spectra were taken and continually compared to spectra taken during the course of the experiment in order to monitor the cleanliness of the sample. The direction of the crystal was obtained prior to the experiment by Laue diffraction, with directional marks made o. the crystal. The crystal was placed normal to the analyzer with incoming photons at an angle of $45^{\circ}$ from the surface. Crystal positioning was done by observing the symmetry of the Fermi surface map obtained from angular distribution patterns taken of the valence band photoelectrons.

An ellipsoidal mirror electron analyzer ${ }^{12}$ located at Brookhaven National Laboratory on the IBM U8 VUV beamline was used to obtain the data in this experiment. In this arrangement, the sample is placed at one focus of an ellipse and the detector behind the other. An ellipsoidal electrostatic mirror faces both the sample and the d-tector and is used as a low-pass filter. A hemisperical grid in front of the detector acts as a high-pass filter while another in front of the sample is used for retarding. The detector consists of microchannel plates and a phosphor screen with a video camera placed behind the screen measuring the electron intensity and digitizing it at a rate of thirty frames per second. The acceptance angle of the analyzer is $\sim 84^{\circ}$, with a typical energy resolution of $0.3 \mathrm{eV}$ and angular resolution of $1 / 4^{\circ}$. For our display measurements, we operated in the constant initial state mode, in which electrons of a particular energy are monitored during the data acquisition. The photon energy was changed when photoelectrons of another wavenumber were desired, along with the repositioning of the analyzer at the new photoelectron energy.

Electron emission patterns of the $P t 4 f_{5} 2$ photoemission peak were measured at equally spaced wavenumbers from $\mathrm{k}=6.0 \AA^{-1}$ to $12.0 \AA^{-1}$. Background spectra were taken at the high energy side of the photoelectron peak and used for normalization. We found that the backgrounds were interchangeable and it was sufficient to measure a few at well chosen energies. Nine images were taken at the first sample position with wavenumber separation $0.6 \AA^{-1}$ and range $7.8 \AA^{-1}$ to $12.0 \AA^{-1}$. The sample was then removed, cleaned and replaced in the same position. In the second position 24 more patterns were obtained, giving a total range of $6.0 \AA^{-1}$ to $12.0 \AA^{-1}$ with a step size of $0.2 \AA^{-1}$.

The relevant holographic oscillatory electron intensity information is separated from the total intensity collected from the analyzer by a three-step process? ${ }^{7}$. First, a division by the background is done to remove the intensity due to electrons in the background and also to normalize the angular through-put function of the analyzer. Next, the electrons are mapped from angular space to a momentum-space counterpart in $\mathrm{k}_{\mathrm{x}}$ and $\mathrm{k}_{\mathrm{y}}$. Finally. the data set is convoluted with a gaussian, the result of which acts as a low-pass filter when subsequently removed from the pattern by a second division. The result of these processes leaves the electron intensity interference pattern which is used to create the real-space holographic images.

\section{RESULTS AND DISCUSSION}

Angstrom scale images have been obtained of atoms in the surface layers of a bulk platinum (111) sample. These images are represented in two ways, using either two or three dimensions. The two dimensional real-space images of atomic positions around the emitter are achieved by application of a two-demensional Fourier transform to the pattern. Prior to transformation, the pattern is phased for a particular distance above the emitter by multiplying by $e^{i k z} \cdot Z$. In the twodimensional representation, slices are taken of a cubic volume perpendicular to the axes. Thi plots used here are generally density plots, where the highest intensity areas represent atomic positions. Three-dimensional representation of the results is done by plotting isosurfaces, or the higher intensity areas, in a volume space which may then be observed from any external point of view.

In figure 1 is shown the real-space $X-Y$ slice taken at $Z=2.2 \AA$, approximately one atomic layer above the emitter. It was obtained from a Pt $4 f_{5 / 2}$ electron intensity pattern of $351 \mathrm{eV}$. This particular image has been squared to highlight the atom positions and v/as derived from a pattern which had been rotationally three-fold symmetry-averaged to offset effects of polarization and to increase clarity. The atoms are close to the actual expected bulk positions, where neighbors in this plane should be separated by $2.56 \AA$. 


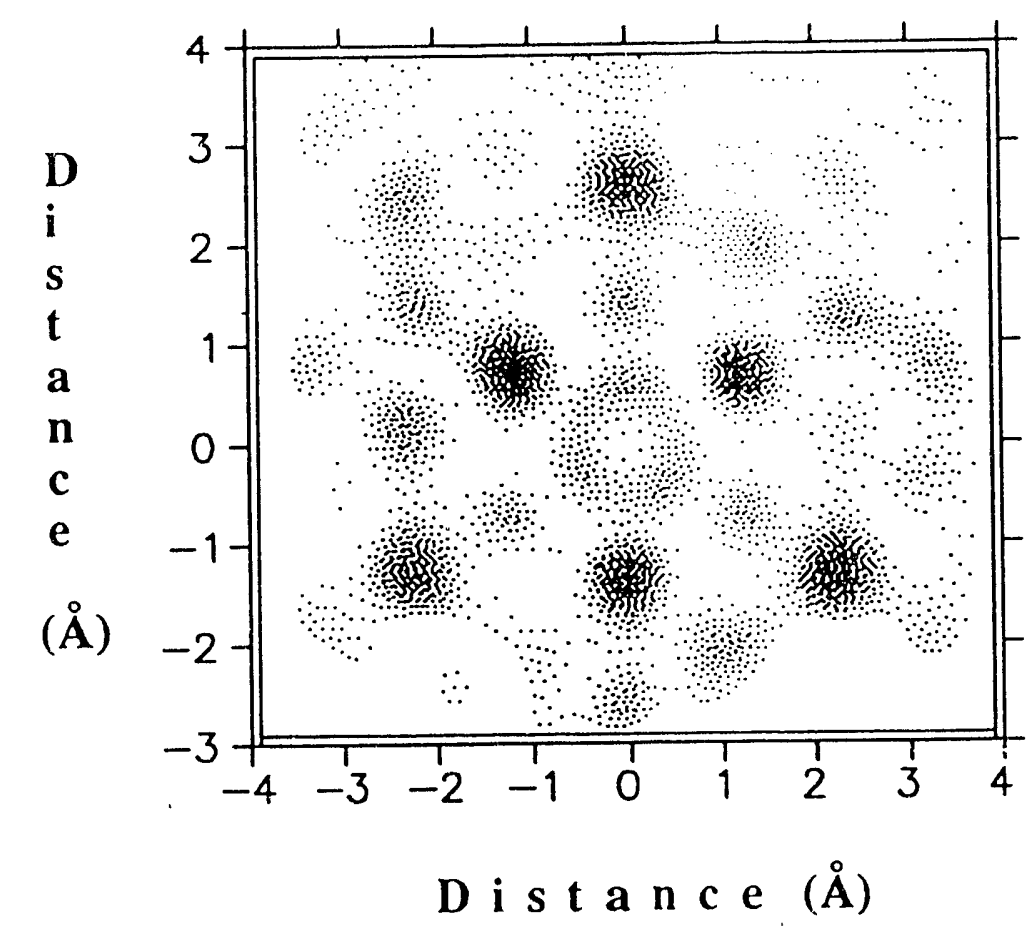

Figure 1. Two-dimensional symmetry-averaged X-Y slice at $Z=2.2 \AA$, one atomic layer above the emitter. Atom positions are shown as dark areas and neighbors should be $2.56 \AA$ apart. This result was obtained from an electron emission pattern of $\mathrm{Pt} 4 \mathrm{f}_{5 / 2}$ photoelectrons with a kinetic energy of $351 \mathrm{eV}$.

Figure 2 shows an iso-density three-dimensional image of atoms above the emitter, which is also sirown at the center of the grid. Three atoms are seen in proper positions one layer above the emitter, three more two layers above, and one three layers up, directly above the emitter. It was generated by phase-summing eight patterns together with equally spaced wavenumbers from $k=8.8 \AA^{-1}$ to $k=10.2 \AA^{-1}$. This result also arises from a pattern which had been-rotationally threefold symmetry-averaged. Notice here that although there is some improvement in Z-resolution, the main effect is a loss of all twin images and artifacts. We note that all of the information shown here has been averaged rotationally for ease of presentation, and that most of the information can be realized without averaging. Variations in position intensity for different directions due to final state wave effects encourage this method of presentation. ${ }^{13}$

\section{CONCLUSIONS}

We have shown that it is possible to image atomic positions of a platinum (111) single-crystal surface using photoelectron holography. Images have been obtained in both single and multiple wavenumber analyses, and the results of the experiments determine structures which agree quantitatively with the positions known from bulk measurements. Because of this method of analysis, fewer transform artifacts are present, atoms are seen beyond nearest neighbors, and effects of photon polarization are observed. When several interference patterns corresponding to different wavenumbers are combined, the results show even fewer artifacts, improved resolution along the $\mathrm{Z}$-direction, and substantial reduction of twin images. All of these factors combined allow the employment of a three-dimensional means of presentation that help in understanding 


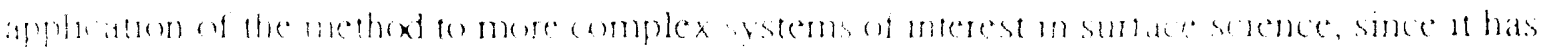

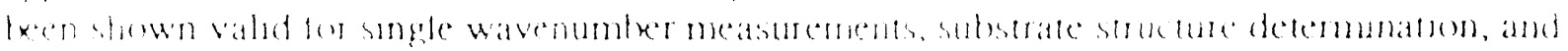

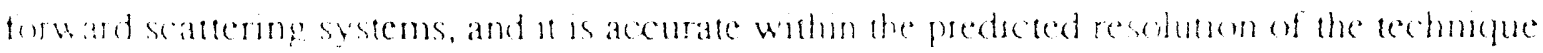

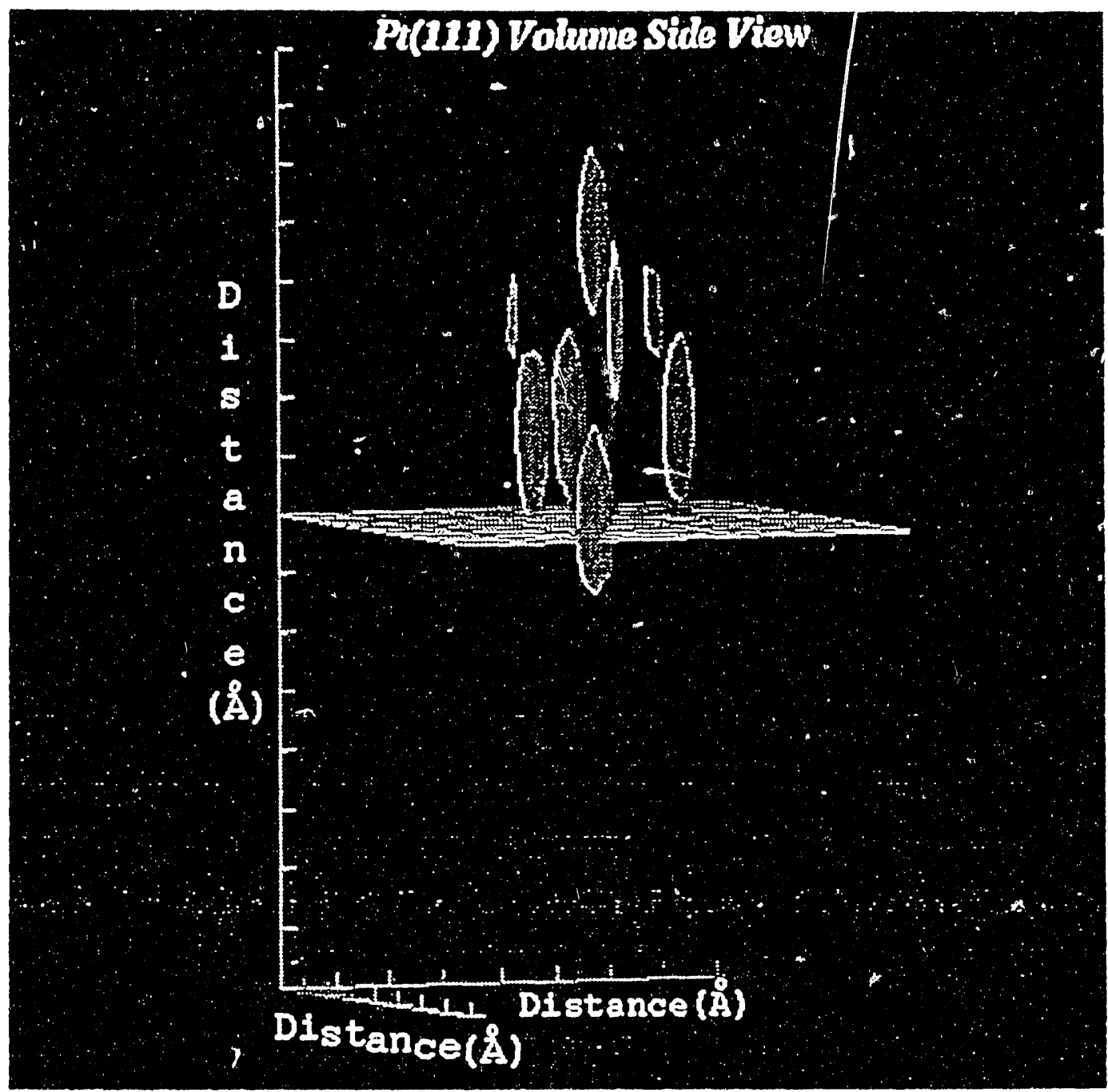

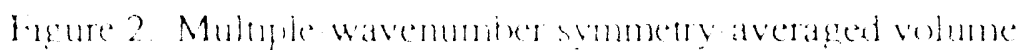

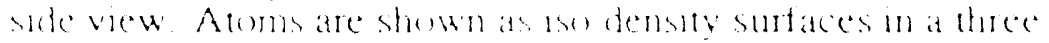

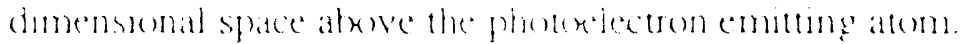

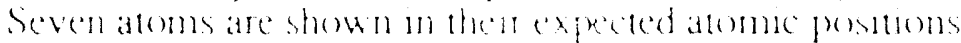

* This work was performed under the auspices of the U.S. Department of Energy by Lawrence I ivermore National Laboratory under contract No. W-7405-tng-48. RIIRIVI I S

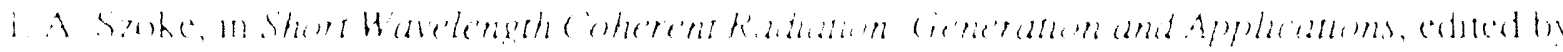

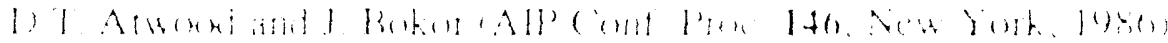

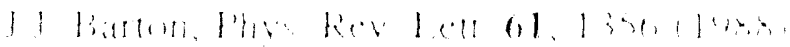

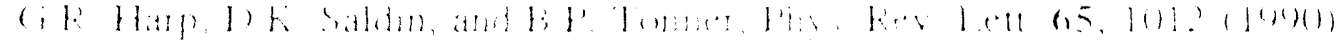

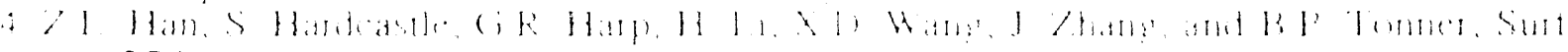
$258 ; 1 ; 1 ; !)$

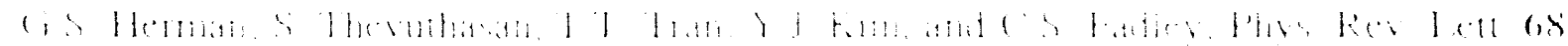

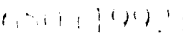

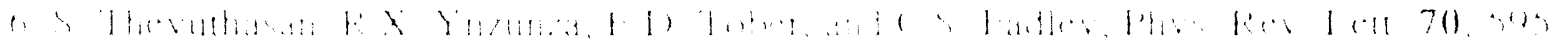
$1,1,1$

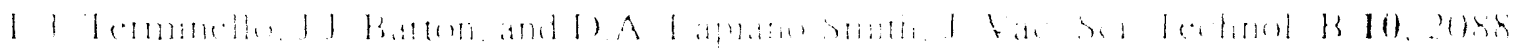

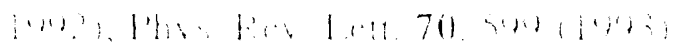

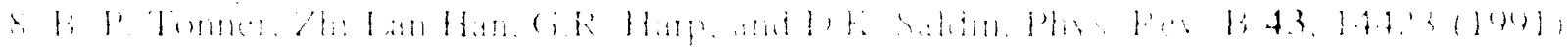


9. H. Huang, Hua Li, and S.Y. Tong, Phys Rev. B 44, 3240 (1990).

10. S.Y. Tong, C.M. Wei, T.C. Zhao, H. Huang, and H. Li, Phys. Rev. Lett. 66, 60 (1991).

11. J.J. Barton, Phys. Rev. Lett. 67, 3106 (1991).

12. D.E. Eastman, J.J. Donelon, N.C. Hien, and F.J. Himpsel, Nuci. Instrum. Methods 172, 327 (1980).

13. B.L. Petersen, L.J. Terminello, J.J. Barton, D.A. Shirley (to be published). 

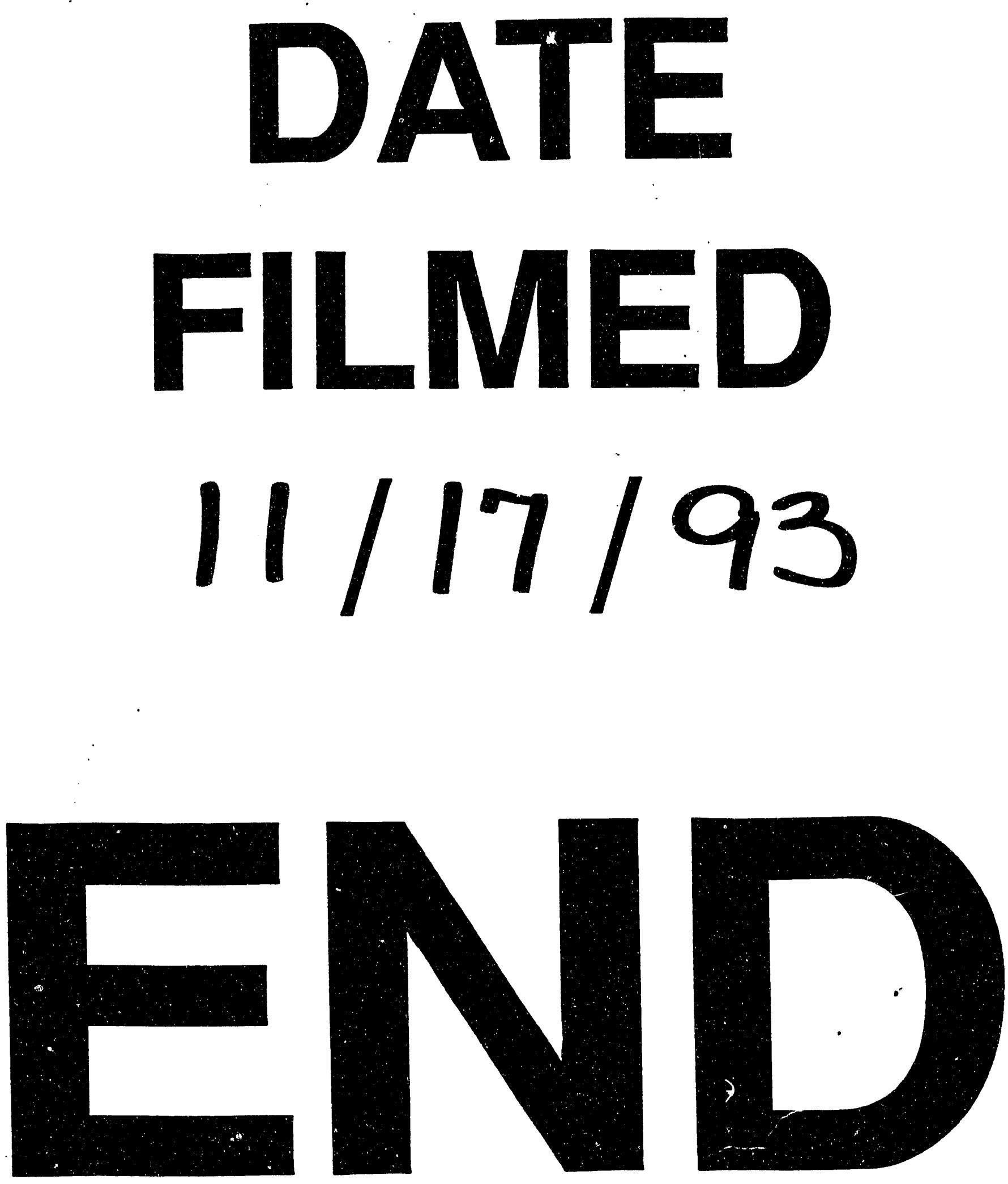
